\title{
INFERENTIAL PROCEDURES BASED ON THE DOUBLE BOOTSTRAP FOR LOG LOGISTIC REGRESSION MODEL WITH CENSORED DATA
}

\author{
Loh Yue Fang ${ }^{1 *}$, Jayanthi Arasan ${ }^{1,2}$, Habshah Midi ${ }^{1,2}$ and Mohd Rizam Abu Bakar ${ }^{1,2}$ \\ ${ }^{1}$ Dept. of Mathematics, Faculty of Science, Universiti Putra Malaysia, 43400 UPM Serdang, Malaysia. \\ ${ }^{2}$ Institute for Mathematical Research, Universiti Putra Malaysia, 43400 UPM Serdang, Malaysia. \\ *Corresponding Author: yuefangloh@yahoo.com
}

\begin{abstract}
Traditional inferential procedures based on the asymptotic normality assumption such as the Wald often produce misleading inferences when dealing with censored data and small samples. Alternative estimation techniques such as the jackknife and bootstrap percentile allow us to construct the interval estimates without relying on any classical assumptions. Recently, the double bootstrap became preferable as it is not only free from any classical assumptions, but also has higher order of accuracy. In this paper, we compare the performances of the interval estimates based on the double bootstrap without pivot with the Wald, jackknife and bootstrap percentile interval estimates for the parameters of the log logistic model with right censored data and covariates via a coverage probability study. Based on the results of the study, we concluded that the double bootstrap without pivot technique works better than the other interval estimation techniques, even when sample size is 25 . The double bootstrap without pivot technique worked well with real data on hypernephroma patients.
\end{abstract}

(Keywords: censored data, coverage probability study, double bootstrap, interval estimation, log logistic)

\section{INTRODUCTION}

In survival analysis, large sample interval estimation based on the asymptotic normality assumptions (also known as Wald interval) is often used as a technique to construct confidence interval for parameters when censored data are involved. Literature shows that misleading inferences could arise when dealing with censored observations and small sample sizes, see [1], [2], [3], [4] and [5]. Jackknife and bootstrap are resampling techniques that do not rely on asymptotic normality assumption and therefore are able to produce more reliable interval estimates.

Jackknife technique was first introduced for bias estimation of a statistic of interest in small sample [6], [7]. The technique later was expanded for estimating standard errors and constructing reasonably reliable confidence interval estimates [8]. A complete review on the jackknife technique and its application can be found in [9]. The bootstrap technique was first introduced by Efron [10] who proposed the bootstrap percentile (B-p) interval. It constructs interval estimates based on the percentiles of the bootstrap distribution of a statistics.

The B-p interval estimation technique is popular due to its simplicity and ability to produce accurate results without having the knowledge of normalizing transformations [11]. More discussion on bootstrap confidence intervals can be obtained in [12], [13], [14], [15] and [16].

Double bootstrap (DB) is a sampling procedure that involves resampling from the bootstrap samples. This technique was originally suggested by Efron [17]. The theory of DB confidence intervals was developed by Hall [18], Beran [19], Loh [20] and Martin [21]. Theoretically DB produces better interval estimation than ordinary or single bootstrap as it has higher order of accuracy. DB reduces the error in the ordinary bootstrap by estimating an error and using it to adjust the ordinary bootstrap according to [22] and [23]. Chernick [24] provided a complete literature on bootstrap interval estimation techniques. The application of the DB confidence interval involving censored and truncated data can be found in [25]. The authors compared the performance of single and double bootstrap interval estimation techniques and highlighted that the latter works better.

In this research, we investigate the performance of the Wald, jackknife, B-p and DB without pivot (DB-p) techniques via coverage probability study at two nominal error probability levels for the parameters of $\log$ logistic regression model with right censored data. Moderate and small samples will be focused in our study. Each sample will be investigated at two 
censoring proportions. A real data set is analyzed and some concluding remarks are made at the end of this paper.

\section{THE MODEL}

The log logistic is a popular parametric distribution in the survival analysis due to its ability to accommodate nonmonotonic hazard functions. Examples of nonmonotonic hazard function are given in the lung cancer trial [26], the curability of breast cancer study [27] and the AIDS infection rate study [28]. Several authors have discussed the log logistic distribution. Bennett [29] presented the linear model for the log odds on survival. Other authors that also made contribution are [30], [31], [32], [33] and [25].

Let $T$ be a random variable representing the lifetimes that follow the log logistic distribution, $\mathbf{x}^{\prime}=\left(x_{0}, x_{1}, \ldots\right.$, $\left.x_{p}\right)$ be the vector of covariates where $x_{0}=1, \boldsymbol{\beta}^{\prime}=\left(\beta_{0}, \beta_{1}\right.$ $\ldots, \beta_{p}$ ) be the vector of unknown parameters and $\sigma>0$. If $Y=\log (T)$ and $z=\left(y-\boldsymbol{\beta}^{\prime} \mathbf{x}\right) / \sigma$ the probability density and survival functions could be expressed respectively as

$$
\begin{gathered}
f(t, \mathbf{x}, \boldsymbol{\beta}, \sigma)=\frac{1}{\sigma} \exp (z)[1+\exp (z)]^{-2}, \\
S(t, \mathbf{x}, \boldsymbol{\beta}, \sigma)=[1+\exp (z)]^{-1}
\end{gathered}
$$

There are both censored and uncensored lifetimes for $i$ $=1,2, \ldots, n$ observations and $p$ covariates. The indicator variable $\delta_{i}$ is equal to 1 when $t_{i}$ is uncensored and 0 when $t_{i}$ is censored.

The likelihood function for the full sample of $n$ independent observations of time and $p$ covariates is,

$$
\begin{aligned}
L(\boldsymbol{\beta}, \sigma)= & \prod_{i=1}^{n}\left(\frac{1}{\sigma} \exp \left(z_{i}\right)\left[1+\exp \left(z_{i}\right)\right]^{-2}\right)^{\delta_{i}} \times \\
& \left(\left[1+\exp \left(z_{i}\right)\right]^{-1}\right)^{1-\delta_{i}} .
\end{aligned}
$$

The log-likelihood function is given as follows,

$$
\begin{aligned}
\ell(\boldsymbol{\beta}, \sigma)= & \sum_{i=1}^{n}\left\{\delta _ { i } \left(-\log \sigma-2 \log \left[1+\exp \left(z_{i}\right)\right]\right.\right. \\
& \left.\left.+z_{i}\right)-\left(1-\delta_{i}\right) \log \left[1+\exp \left(z_{i}\right)\right]\right\} .
\end{aligned}
$$

Let us define $A_{i}=\exp \left(z_{i}\right)\left[1+\exp \left(z_{i}\right)\right]^{-1}$, where $i=1,2$, $\ldots, n$. For $j, k=0,1, \ldots, p$ and $x_{i 0}=1$, the first and second derivatives of the log-likelihood function are,

$$
\frac{\partial \ell(\boldsymbol{\beta}, \sigma)}{\partial \beta_{j}}=\sum_{i=1}^{n} \frac{x_{i j}}{\sigma}\left\{-\delta_{i}+\left(1+\delta_{i}\right) A_{i}\right\},
$$

$$
\begin{aligned}
\frac{\partial \ell(\boldsymbol{\beta}, \sigma)}{\partial \sigma}= & \sum_{i=1}^{n} \frac{1}{\sigma}\left\{-\delta_{i}\left(1+z_{i}\right)+\left(1+\delta_{i}\right) z_{i} A_{i}\right\}, \\
\frac{\partial^{2} \ell(\boldsymbol{\beta}, \sigma)}{\partial \beta_{j} \partial \sigma}= & \sum_{i=1}^{n} \frac{x_{i j}}{\sigma}\left\{-\delta_{i}\left(\frac{1}{\sigma}\right)+\left(1+\delta_{i}\right) A_{i} \times\right. \\
& {\left.\left[1+z_{i}\left(1+A_{i}\right)\right]\right\}, } \\
\frac{\partial^{2} \ell(\boldsymbol{\beta}, \sigma)}{\partial \beta_{j} \partial \beta_{k}}= & \sum_{i=1}^{n} \frac{x_{i j} x_{i k}}{\sigma^{2}}\left(1+\delta_{i}\right) A_{i}\left(A_{i}-1\right), \\
\frac{\partial^{2} \ell(\boldsymbol{\beta}, \sigma)}{\partial \sigma^{2}}= & \sum_{i=1}^{n} \frac{1}{\sigma^{2}}\left\{-\delta_{i}\left(1+2 z_{i}\right)-\left(1+\delta_{i}\right) \times\right. \\
& \left.z_{i}^{2} A_{i}\left(1+\frac{1}{\sigma^{2}} A_{i}\right)\right\} .
\end{aligned}
$$

\section{CONFIDENCE INTERVAL ESTIMATION PROCEDURES}

In this section, we discuss Wald, jackknife, B-p and DB-p techniques for constructing the confidence interval for the parameters of the log logistic regression model in the presence of right censored data. We consider one covariate in the model. The model involves three parameters, $\beta_{0}, \beta_{1}$ and $\sigma$. The bootstrap samples were generated using the nonparametric bootstrap resampling technique.

\section{Asymptotic Normality (Wald)}

Let $\hat{\boldsymbol{\theta}}$ be the maximum likelihood estimate (MLE) for the vector of parameters $\boldsymbol{\theta}$. Cox and Hinkley [34] showed that under mild regularity conditions, $\hat{\boldsymbol{\theta}}$ is asymptotically normally distributed with mean $\boldsymbol{\theta}$ and covariance matrix $I(\boldsymbol{\theta})^{-1}$, where $I(\boldsymbol{\theta})$ is the Fisher information matrix evaluated at $\boldsymbol{\theta}$. The matrix $I(\theta)$ can be estimated by the observed information matrix $i(\hat{\boldsymbol{\theta}})$. The estimate of $\operatorname{var}\left(\hat{\theta}_{j}\right)$ is then given by the $j^{\text {th }}$ diagonal element of the inverse of $i(\hat{\boldsymbol{\theta}})$. If $z_{1-\alpha / 2}$ is the $1-\alpha / 2$ quantile of the standard normal distribution, then the $100(1-\alpha) \%$ confidence interval for $\theta_{j}$ could be expressed as

$$
\hat{\theta}_{j} \pm z_{1-\frac{\alpha}{2}} \sqrt{\hat{\operatorname{var}\left(\hat{\theta}_{j}\right)}}
$$

\section{Jackknife}

The jackknife is a resampling technique where each subsample removes one observation from the original sample [11]. If we have a sample $\mathbf{y}=\left(y_{1}, y_{2}, \ldots, y_{n}\right)$, 
then the $i^{\text {th }}$ jackknife sample will be $\mathbf{y}_{(i)}=\left(y_{1}, y_{2}, \ldots, y_{i-1}\right.$, $y_{i+1}, \ldots, y_{n}$ ) for $i=1,2, \ldots, n$. Let $\hat{\theta}$ be the MLE for parameter $\theta$, then $\hat{\theta}_{(i)}$ will be the jackknife replication of $\hat{\theta}$ obtained from the $i^{\text {th }}$ jackknife sample. The jackknife estimate of the parameter $\theta$ and jackknife estimate of standard error is then calculated by using

$$
\begin{aligned}
& \hat{\theta}_{\text {jack }}=\hat{\theta}-(n-1)\left(\hat{\theta}_{(\cdot)}-\hat{\theta}\right), \\
& \hat{\operatorname{se}}(\hat{\theta})_{\text {jack }}=\left[\frac{n-1}{n} \sum_{i=1}^{n}\left(\hat{\theta}_{(i)}-\hat{\theta}_{(\cdot)}\right)\right]^{1 / 2} \text {, }
\end{aligned}
$$

where $\hat{\theta}_{(\cdot)}=\sum_{i=1}^{n} \hat{\theta}_{(i)} / n$.

If $t_{(1-\alpha / 2, n-1)}$ is the $1-\alpha / 2$ quantile of the student's $t$ distribution at $n-1$ degrees of freedom, then the 100(1 $-\alpha) \%$ jackknife confidence interval for $\theta$ could be expressed as

$$
\hat{\theta}_{\mathrm{jack}} \pm t_{\left(1-\frac{\alpha}{2}, n-1\right)} \hat{\operatorname{se}(\hat{\theta})_{\mathrm{jack}}}
$$

\section{Bootstrap Percentile}

The B-p interval is constructed from the percentiles of the bootstrap distribution of the MLE for parameter $\theta$. For the sample $\mathbf{y}=\left(y_{1}, y_{2}, \ldots, y_{n}\right)$, we first generate $B$ independent bootstrap samples, $\mathbf{y}^{*_{1}}, \mathbf{y}^{{ }^{* 2}}, \ldots, \mathbf{y}^{{ }^{*} B}$. Then we compute the MLE, $\hat{\theta}_{b}^{*}$ from the bootstrap sample, $\mathbf{y}^{* b}$ for $b=1,2, \ldots, B$. Following that, we sort the $\hat{\theta}_{b}^{*}$ in the ordered list, which we will have $\hat{\theta}_{[1]}^{*}, \hat{\theta}_{[2]}^{*}, \ldots, \hat{\theta}_{[B]}^{*}$. The $100(1-\alpha) \%$ bootstrap percentiles confidence interval for $\theta$ could be expressed as

$$
\hat{\theta}_{[B \cdot(\alpha / 2)]}^{*}<\hat{\theta}<\hat{\theta}_{[B \cdot(1-\alpha / 2)]}^{*} .
$$

The value of $B$ is chosen such that values of $B \cdot(\alpha / 2)$ and $B \cdot(1-\alpha / 2)$ are integers.

\section{Double Bootstrap without Pivot}

The DB-p confidence interval was proposed by Shi [35] and discussed by Letson and McCullough [23]. This technique is particularly useful when pivotal information is not available. The steps to obtain the DB-p confidence interval for parameter $\theta$ are as follows,

1. Obtain the MLE $\hat{\theta}$ based on the sample $\mathbf{y}=\left(y_{1}, y_{2}\right.$, $\left.\ldots, y_{n}\right)$.
2. Generate $B$ independent bootstrap samples, $\mathbf{y}^{{ }^{* 1}}, \mathbf{y}^{{ }^{* 2}}$, $\ldots, \mathbf{y}^{* B}$ from the original sample.

3. Compute $\hat{\theta}_{b}^{*}$, MLE from the bootstrap sample, $\mathbf{y}^{* b}$ for $b=1,2, \ldots, B$.

4. Sort the $\hat{\theta}_{b}^{*}$ in an ascending order to obtain $\hat{\theta}_{[1]}^{*}$, $\hat{\theta}_{[2]}^{*}, \ldots, \hat{\theta}_{[B]}^{*}$.

5. Draw another $C$ bootstrap resamples for each $\mathbf{y}^{* b}, b$ $=1,2, \ldots, B$.

6. Compute the $\hat{\theta}_{b c}^{* *}$, MLE from the bootstrap resample for $b=1,2, \ldots, B$ and $c=1,2, \ldots, C$.

7. For each $c$, calculate the total number of $\hat{\theta}_{b c}^{* *}$ that is less than or equal to $\hat{\theta}$ and divide by numbers of bootstrap resamples, $C$,

$$
Q_{b}=\frac{\#\left(\hat{\theta}_{b c}^{* *} \leq \hat{\theta}\right)}{C} .
$$

8. Sort the $Q_{b}$ in an ascending order to obtain $Q_{[1]}$, $Q_{[2]}, \ldots, Q_{[B]}$.

9. Calculate the values, $l=B \cdot Q_{[B \cdot(\alpha / 2)]}$ and $u=B \cdot Q_{[B \cdot(1}$ $-\alpha / 2)]$.

10. The $100(1-\alpha) \%$ DB-p confidence interval for parameter $\theta$ will be

$$
\hat{\theta}_{[l]}^{*}<\hat{\theta}<\hat{\theta}_{[u]}^{*} .
$$

\section{COVERAGE PROBABILITY STUDY}

\section{Study Design}

We conducted a simulation study with $N=1000$ for sample sizes, $n=25,30,40,50$ and 60 to compare the performance of the confidence interval estimates at two nominal error probabilities, $\alpha=0.05$ and 0.10 . Two levels of approximate censoring proportions $(\mathrm{cp})=5 \%$ and $10 \%$ were used. We generated observations from the $\log$ logistic distribution with $4.4,-0.03$ and 0.6 as the true values for parameters $\beta_{0}, \beta_{1}$ and $\sigma$ respectively to replicate data usually seen in the lung cancer studies [26].

The covariate $x_{i}$ were generated from $N(0,1)$. We generated random numbers $u_{i}$ from $U(0,1)$ to produce $t_{i}$ where $t_{i}=\exp \left\{\beta_{0}+\beta_{1} x_{i}+\sigma \log \left[\left(1-u_{i}\right) / u_{i}\right]\right\}$. In order to obtain censored observations, we simulate the censoring time, $c_{i} \sim \exp (\lambda)$ where the value of $\lambda$ can be adjusted to obtain the desired approximate censoring proportion in our data. We generated $B=1000$ bootstrap samples to construct the B-p and DB-p intervals. $C=150$ bootstrap resamples were generated from each bootstrap sample for the DB-p interval. 
The coverage probability error of a confidence interval is the probability that the true value of the parameter falls outside the interval. We obtained the estimated coverage probability error by summing up the numbers of interval that did not contain the true parameter value divided by the total number of samples. The estimated left (right) error probability was obtained by summing up the numbers for the left (right) endpoint was more (less) than the true parameter value divided by the total number of samples. We calculated the left, right and total error probabilities for each parameter.

As given in [36], if $\hat{\alpha}$ is the estimated coverage error probability, then its standard error is approximately $\operatorname{se}(\hat{\alpha})=[\alpha(1-\alpha) / N]^{1 / 2}$. The interval is called anticonservative if the total error probability is more than $\alpha+2.58 \mathrm{se}(\hat{\alpha})$. If the total error probability is less than $\alpha-2.58 \operatorname{se}(\hat{\alpha})$, the interval is called conservative. The interval is called symmetric when the larger of the left or right error probability is less than 1.5 times the smaller one. The simulation study was performed using the $\mathrm{R}$ programming language.

\section{Study Results and Discussions}

The performances of these interval estimation techniques were evaluated based on the total numbers of anticonservative (AC), conservative (C) and asymmetrical (AS) intervals. When $\alpha=0.05$, at $n \leq 30$, the DB-p and jackknife techniques performed the best compared to the Wald and B-p (Table 1). The DB-p is slightly better than the jackknife as it produces the least numbers of $\mathrm{AC}$ and $\mathrm{AS}$ intervals. However, it produces $C$ interval when the data are censored. When $n>30$, the DB-p performs the best followed by the jackknife, B-p and Wald as it did not produce any $\mathrm{AC}$ or $\mathrm{C}$ intervals.

When $\alpha=0.10$, the DB-p performs the best even at $n \leq$ 30 because it does not produce any $\mathrm{AC}, \mathrm{C}$ or $\mathrm{AS}$ intervals (Table 2). This is followed by the jackknife, Wald and B-p where these techniques are still producing AC and AS intervals. When $n>30$, the DB$\mathrm{p}$ technique is the preferred technique as it only produces one AC interval, followed by the jackknife, Wald and B-p.

Hence, overall the DB-p performs the best at all nominal levels, sample sizes and censoring proportions. The jackknife performs well by producing few AC intervals. The B-p and Wald techniques did not perform well as they produced too many AC and AS intervals. In addition, the left and right error probabilities for DB$\mathrm{p}$ technique are much closer to the $\alpha / 2$ as compared to the Wald, jackknife and B-p techniques. The summary of interval estimation at $\alpha=0.05$ and 0.10 for each parameter is given in Table 3 and Table 4. An example of graphical display of the left and right error probabilities for the interval estimation techniques is given in Figure 1-3.

Table 1. Performance of the interval estimation techniques at different $n$ for $\alpha=0.05$

\begin{tabular}{|l|l|ccc|ccc|ccc|}
\hline \multirow{2}{*}{$\boldsymbol{n}$} & \multirow{2}{*}{ Technique } & \multicolumn{3}{|c|}{ Uncensored } & \multicolumn{4}{|c|}{$\mathbf{c p}=\mathbf{5 \%}$} & \multicolumn{3}{c|}{$\mathbf{c p}=\mathbf{1 0 \%}$} \\
\cline { 3 - 10 } & & AC & C & AS & AC & C & AS & AC & C & AS \\
\hline 25 & Wald & 3 & 0 & 1 & 3 & 0 & 1 & 2 & 0 & 1 \\
& Jackknife & 2 & 0 & 1 & 1 & 0 & 2 & 1 & 0 & 1 \\
& B-p & 1 & 0 & 2 & 1 & 0 & 2 & 1 & 0 & 2 \\
& DB-p & 1 & 0 & 1 & 1 & 1 & 1 & 1 & 1 & 1 \\
\hline 30 & Wald & 3 & 0 & 1 & 3 & 0 & 1 & 3 & 0 & 1 \\
& Jackknife & 0 & 0 & 1 & 1 & 0 & 1 & 0 & 0 & 1 \\
& B-p & 2 & 0 & 1 & 2 & 0 & 2 & 2 & 0 & 2 \\
& DB-p & 0 & 0 & 1 & 0 & 0 & 1 & 0 & 0 & 1 \\
\hline 40 & Wald & 1 & 0 & 1 & 1 & 0 & 1 & 1 & 0 & 2 \\
& Jackknife & 0 & 0 & 1 & 1 & 0 & 1 & 1 & 0 & 2 \\
& B-p & 2 & 0 & 1 & 1 & 0 & 1 & 1 & 0 & 1 \\
& DB-p & 0 & 0 & 0 & 0 & 0 & 0 & 0 & 0 & 0 \\
\hline 50 & Wald & 2 & 0 & 1 & 2 & 0 & 1 & 1 & 0 & 1 \\
& Jackknife & 0 & 0 & 2 & 0 & 0 & 1 & 0 & 0 & 1 \\
& B-p & 1 & 0 & 1 & 1 & 0 & 1 & 1 & 0 & 2 \\
& DB-p & 0 & 0 & 0 & 0 & 0 & 0 & 0 & 0 & 1 \\
\hline 60 & Wald & 1 & 0 & 2 & 1 & 0 & 2 & 1 & 0 & 3 \\
& Jackknife & 0 & 0 & 2 & 0 & 0 & 1 & 0 & 0 & 2 \\
& B-p & 1 & 0 & 1 & 1 & 0 & 1 & 1 & 0 & 1 \\
& DB-p & 0 & 0 & 1 & 0 & 0 & 1 & 0 & 0 & 1 \\
\hline
\end{tabular}


Malaysian Journal of Science 34 (2) : 199-207 (2015)

Table 2. Performance of the interval estimation techniques at different $n$ for $\alpha=0.10$

\begin{tabular}{|l|l|ccc|ccc|ccc|}
\hline \multirow{2}{*}{$n$} & \multirow{2}{*}{ Technique } & \multicolumn{3}{|c|}{ Uncensored } & \multicolumn{3}{|c|}{$\mathbf{c p}=\mathbf{5 \%}$} & \multicolumn{3}{c|}{$\mathbf{c p}=\mathbf{1 0 \%}$} \\
\cline { 2 - 10 } & & AC & $\mathbf{C}$ & $\mathbf{A S}$ & $\mathbf{A C}$ & $\mathbf{C}$ & $\mathbf{A S}$ & $\mathbf{A C}$ & $\mathbf{C}$ & $\mathbf{A S}$ \\
\hline 25 & Wald & 2 & 0 & 1 & 2 & 0 & 1 & 2 & 0 & 1 \\
& Jackknife & 1 & 0 & 1 & 1 & 0 & 1 & 0 & 0 & 1 \\
& B-p & 1 & 0 & 1 & 1 & 0 & 1 & 1 & 0 & 2 \\
& DB-p & 0 & 0 & 0 & 0 & 0 & 0 & 0 & 0 & 0 \\
\hline 30 & Wald & 2 & 0 & 1 & 2 & 0 & 1 & 2 & 0 & 1 \\
& Jackknife & 0 & 0 & 1 & 0 & 0 & 1 & 1 & 0 & 1 \\
& B-p & 2 & 0 & 1 & 2 & 0 & 2 & 2 & 0 & 1 \\
& DB-p & 0 & 0 & 0 & 0 & 0 & 0 & 0 & 0 & 0 \\
\hline 40 & Wald & 1 & 0 & 1 & 1 & 0 & 1 & 1 & 0 & 1 \\
& Jackknife & 0 & 0 & 1 & 0 & 0 & 1 & 0 & 0 & 1 \\
& B-p & 1 & 0 & 1 & 1 & 0 & 1 & 1 & 0 & 1 \\
& DB-p & 1 & 0 & 0 & 0 & 0 & 0 & 0 & 0 & 0 \\
\hline 50 & Wald & 0 & 0 & 1 & 1 & 0 & 1 & 0 & 0 & 1 \\
& Jackknife & 0 & 0 & 1 & 0 & 0 & 1 & 0 & 0 & 1 \\
& B-p & 1 & 0 & 1 & 1 & 0 & 1 & 1 & 0 & 1 \\
& DB-p & 0 & 0 & 0 & 0 & 0 & 0 & 0 & 0 & 0 \\
\hline 60 & Wald & 1 & 0 & 2 & 1 & 0 & 1 & 1 & 0 & 1 \\
& Jackknife & 0 & 0 & 2 & 0 & 0 & 1 & 0 & 0 & 1 \\
& B-p & 1 & 0 & 1 & 1 & 0 & 1 & 1 & 0 & 1 \\
& DB-p & 0 & 0 & 0 & 0 & 0 & 0 & 0 & 0 & 0 \\
\hline
\end{tabular}

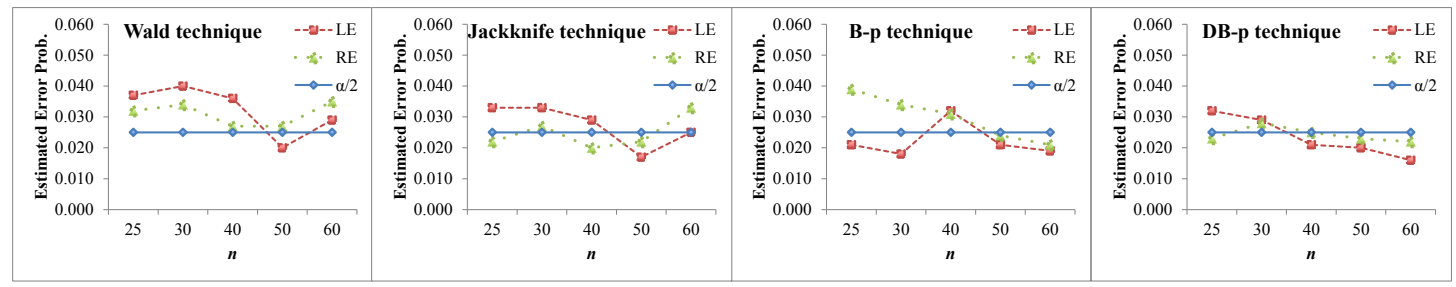

Figure 1. Interval estimates for parameter $\beta_{0}$ at $\alpha=0.05$ and $\mathrm{cp}=5 \%$

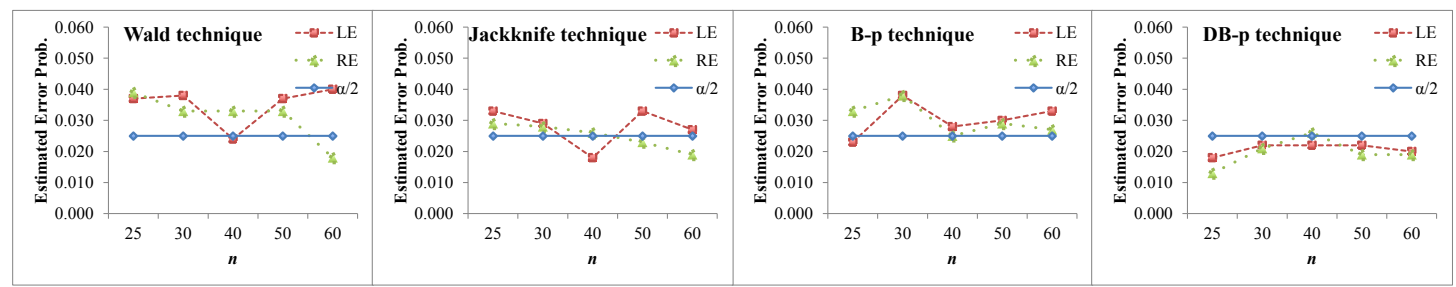

Figure 2. Interval estimates for parameter $\beta_{1}$ at $\alpha=0.05$ and $\mathrm{cp}=5 \%$

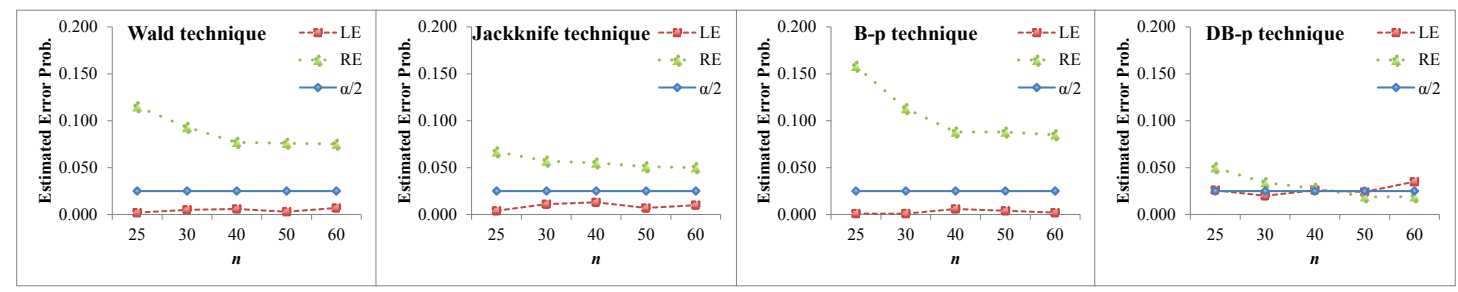

Figure 3. Interval estimates for parameter $\sigma$ at $\alpha=0.05$ and $\mathrm{cp}=5 \%$ 
Table 3. Summary of the performance of the interval estimates for all the parameters at $\alpha=0.05$

\begin{tabular}{|c|c|c|c|c|c|c|c|c|c|c|}
\hline \multirow{2}{*}{ cp } & \multirow{2}{*}{ Technique } & \multicolumn{3}{|c|}{$\boldsymbol{\beta}_{0}$} & \multicolumn{3}{|c|}{$\beta_{1}$} & \multicolumn{3}{|c|}{$\sigma$} \\
\hline & & $\mathbf{A C}$ & C & AS & $\mathrm{AC}$ & $\mathbf{C}$ & AS & $\mathbf{A C}$ & $\mathbf{C}$ & AS \\
\hline \multirow{4}{*}{ 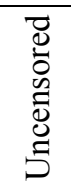 } & Wald & 2 & 0 & 0 & 3 & 0 & 1 & 5 & 0 & 5 \\
\hline & Jackknife & 0 & 0 & 1 & 1 & 0 & 1 & 1 & 0 & 5 \\
\hline & B-p & 1 & 0 & 1 & 1 & 0 & 0 & 5 & 0 & 5 \\
\hline & DB-p & 0 & 0 & 0 & 0 & 0 & 0 & 1 & 0 & 3 \\
\hline \multirow[t]{4}{*}{$5 \%$} & Wald & 2 & 0 & 0 & 3 & 0 & 1 & 5 & 0 & 5 \\
\hline & Jackknife & 0 & 0 & 1 & 0 & 0 & 0 & 3 & 0 & 5 \\
\hline & B-p & 0 & 0 & 2 & 1 & 0 & 0 & 5 & 0 & 5 \\
\hline & DB-p & 0 & 0 & 0 & 0 & 1 & 0 & 1 & 0 & 3 \\
\hline \multirow[t]{4}{*}{$10 \%$} & Wald & 1 & 0 & 1 & 2 & 0 & 2 & 5 & 0 & 5 \\
\hline & Jackknife & 0 & 0 & 1 & 0 & 0 & 1 & 2 & 0 & 5 \\
\hline & B-p & 0 & 0 & 3 & 1 & 0 & 0 & 5 & 0 & 5 \\
\hline & DB-p & 0 & 0 & 0 & 0 & 1 & 0 & 1 & 0 & 3 \\
\hline
\end{tabular}

Table 4. Summary of the performance of the interval estimates for all the parameters at $\alpha=0.10$

\begin{tabular}{|c|c|c|c|c|c|c|c|c|c|c|}
\hline \multirow{2}{*}{ cp } & \multirow{2}{*}{ Technique } & \multicolumn{3}{|c|}{$\beta_{0}$} & \multicolumn{3}{|c|}{$\beta_{1}$} & \multicolumn{3}{|c|}{$\sigma$} \\
\hline & & $\mathrm{AC}$ & C & AS & $\mathrm{AC}$ & C & AS & $\mathrm{AC}$ & C & AS \\
\hline \multirow{4}{*}{ 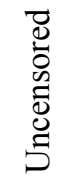 } & Wald & 1 & 0 & 0 & 1 & 0 & 1 & 4 & 0 & 5 \\
\hline & Jackknife & 0 & 0 & 0 & 0 & 0 & 1 & 1 & 0 & 5 \\
\hline & B-p & 0 & 0 & 0 & 1 & 0 & 0 & 5 & 0 & 5 \\
\hline & DB-p & 0 & 0 & 0 & 0 & 0 & 0 & 1 & 0 & 0 \\
\hline \multirow[t]{4}{*}{$5 \%$} & Wald & 1 & 0 & 0 & 2 & 0 & 0 & 4 & 0 & 5 \\
\hline & Jackknife & 0 & 0 & 0 & 0 & 0 & 0 & 1 & 0 & 5 \\
\hline & B-p & 0 & 0 & 1 & 1 & 0 & 0 & 5 & 0 & 5 \\
\hline & DB-p & 0 & 0 & 0 & 0 & 0 & 0 & 0 & 0 & 0 \\
\hline \multirow[t]{4}{*}{$10 \%$} & Wald & 0 & 0 & 0 & 2 & 0 & 0 & 4 & 0 & 5 \\
\hline & Jackknife & 0 & 0 & 0 & 0 & 0 & 0 & 1 & 0 & 5 \\
\hline & B-p & 0 & 0 & 1 & 1 & 0 & 0 & 5 & 0 & 5 \\
\hline & DB-p & 0 & 0 & 0 & 0 & 0 & 0 & 0 & 0 & 0 \\
\hline
\end{tabular}

\section{REAL DATA ANALYSIS}

Survival times of 29 hypernephroma patients with nephrectomy were obtained from [37]. Here, the age group was chosen as the covariate and $13.8 \%$ of the data is censored. Figure 4 shows that the log logistic hazard plot is rather linear, suggesting the log logistic distribution is a reasonable fit to the data. Table 5 gives the parameter estimates when the data were fitted to the $\log$ logistic regression model. The $95 \%$ confidence interval using the Wald, jackknife, B-p and DB-p interval estimation techniques were also given.

Figure 5 gives the histogram of 1000 bootstrap replications of parameter estimates $\hat{\beta}_{0}, \hat{\beta}_{1}$ and $\hat{\sigma}$. The sampling distribution for all parameters are rather skewed and not normally distributed when tested via the Kolmogorov-Smirnov test at $\alpha=0.05$. Therefore, the Wald interval based on the asymptotic normality assumptions of the MLEs might not be reliable and alternative interval estimation may produce more desirable results.

The 95\% DB-p confidence intervals for all parameters are widest compared to other methods because they need to accommodate the bias and adjust the error in the ordinary bootstrap procedure (Table 5). The DB-p interval for $\sigma$ is the narrowest among the three parameters which agrees with the histogram in Figure 5 where bootstrap replication of $\hat{\sigma}$ is less skewed. Although the jackknife, Wald and B-p produce narrower intervals, they may not be reliable as they may fail to include the true parameters value, as indicated by our simulation studies. 


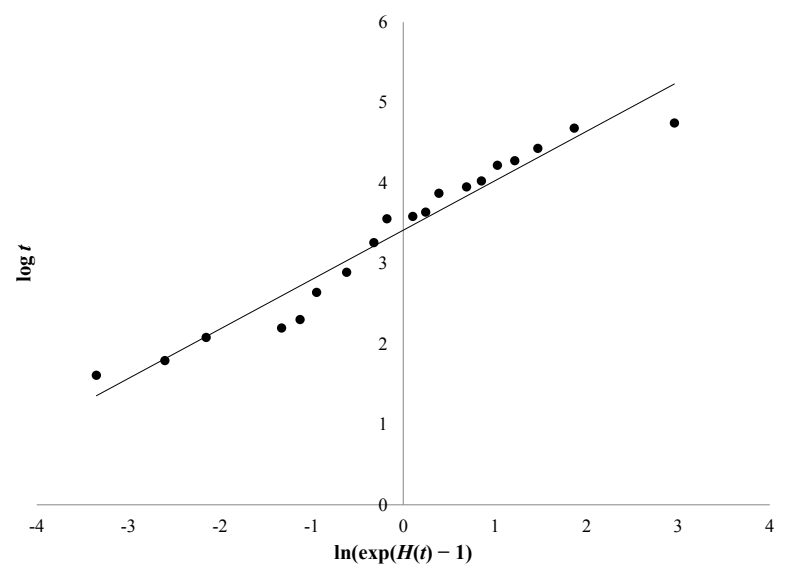

Figure 4. Log logistic hazard plot of the survival time of 29 hypernephroma patients with nephrectomy

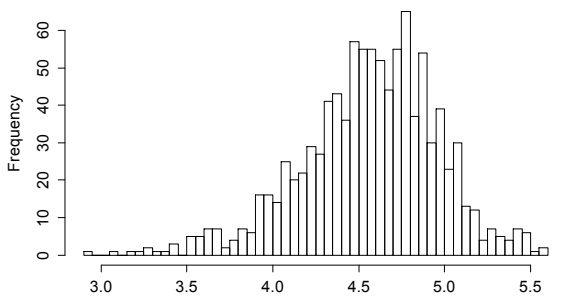

(a) Bootstrap replication of $\hat{\beta}_{0}$

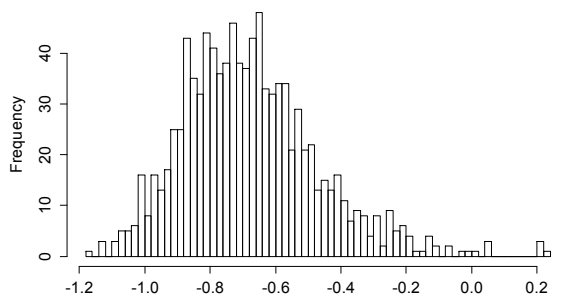

(b) Bootstrap replication of $\hat{\beta}_{1}$

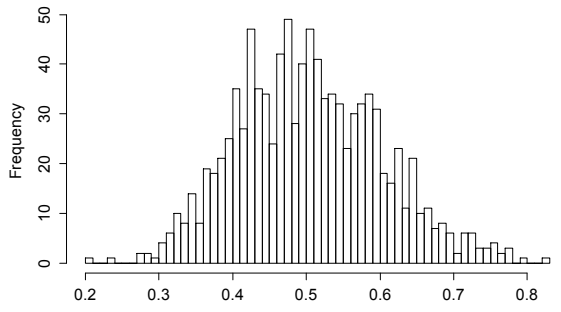

(c) Bootstrap replication of $\hat{\sigma}$

Figure 5. 1000 bootstrap replication of $\hat{\beta}_{0}, \hat{\beta}_{1}$ and $\hat{\sigma}$ for hypernephroma patients with nephrectomy data

Table 5. MLE of hypernephroma patients with nephrectomy data and $95 \%$ confidence intervals

\begin{tabular}{|l|l|l|l|}
\hline $\boldsymbol{\theta}$ & $\beta_{0}$ & $\beta_{1}$ & $\sigma$ \\
\hline$\hat{\boldsymbol{\theta}}$ & 4.58 & -0.69 & 0.53 \\
$\mathbf{s e}$ & 0.397 & 0.221 & 0.089 \\
Wald & $(3.799,5.354)$ & $(-1.125,-0.257)$ & $(0.351,0.700)$ \\
Jackknife & $(3.856,5.388)$ & $(-1.117,-0.326)$ & $(0.330,0.752)$ \\
B-p & $(3.641,5.300)$ & $(-1.020,-0.203)$ & $(0.329,0.715)$ \\
DB-p & $(3.525,5.247)$ & $(-1.041,-0.111)$ & $(0.368,0.865)$ \\
\hline
\end{tabular}




\section{CONCLUSION}

Based on the coverage probability studies, we would recommend the DB-p technique to be employed as the preferred method for the interval estimation for the parameters of log logistic regression model especially when $n$ is small. It works well when the data is either uncensored or censored. This result varies from the result obtained by Arasan and Adam [25] for the model without covariate when data is truncated, which actually did not recommend the DB-p method. The jackknife technique could be used as an alternative method for the model as it performs as good as the DBp. The Wald technique starts to work well when $n>30$, as expected. However, it produces quite a number of anticonservative and asymmetric intervals.

Skewed distributions are common in the survival analysis even when the data is uncensored. When we bootstrap the sample with censored observations, it is possible to have highly skewed bootstrap replications of parameter estimates. In this case, the B-p technique is more likely to fail as it uses the empirical distribution based on the bootstrap replication of the data in hand. The performance of the B-p interval does not seem to be significantly better than the other interval estimation techniques. Therefore, we will not recommend B-p interval for this model.

In this research, we considered only small to moderate samples with low censoring proportions. Larger sample sizes and higher censoring proportions could be considered in future. Other double bootstrap interval estimation techniques such as DB-t can also be considered for the parameters of log logistic regression model in future.

\section{ACKNOWLEDGMENT}

We gratefully acknowledge financial support from the Ministry of Education Malaysia. The research leading to these results has received funding from the Fundamental Research Grant Scheme (FRGS 2014) under vote no. 5524673 .

\section{REFERENCES}

1. Jeng, S.L. and Meeker, W.Q. (2000). Comparisons of approximate confidence interval procedures for type I censored data. Technometrics, vol. 42, no. 2, pp. 135-148.

2. Baklizi, A.S. (2002). Likelihood based estimation in the logistic model with time censored data. Pertanika Journal of Science and Technology, vol. 10, no. 1, pp. 55-62.
3. Arasan, J. (2009). Interval estimation for parameters of a bivariate time varying covariate model. Pertanika Journal of Science and Technology, vol. 17, no. 2, pp. 313-323.

4. Arasan, J. and Lunn, M. (2009). Survival model of a parallel system with dependent failures and time varying covariates. Journal of Statistical Planning and Inference, vol. 139, pp. 944-951.

5. Kiani, K., Arasan, J. and Midi, H. (2012). Interval estimations for parameters of gompertz model with time-dependent covariate and right censored data. Sains Malaysiana, vol. 41, no. 4, pp. 471-480.

6. Quenouille, M.H. (1949). Approximate tests of correlation in time-series. Journal of the Royal Statistical Society. Series B (Methodological), vol. 11 , no. 1, pp. 68-84.

7. Quenouille, M.H. (1956). Notes on bias in estimation. Biometrika, pp. 353-360.

8. Tukey, J.W. (1958). Bias and confidence in notquite large samples. Annals of Mathematical Statistics, vol. 29, no. 2, p. 614.

9. Miller, R.G. (1974). The jackknife - a review. Biometrika, vol. 61, no. 1, pp. 1-15.

10. Efron, B. (1979). Bootstrap methods: Another look at the jackknife. The Annals of Statistics, vol. 7, no. 1, pp. 1-26.

11. Efron, B. and Tibshirani, R.J. (1993). An introduction to the bootstrap, Monographs on Statistics and Applied Probability, vol. 57. Chapman and Hall.

12. Lunneborg, C.E. (1985). Estimating the correlation coefficient: The bootstrap approach. Psychological Bulletin, vol. 98, no. 1, pp. 209-215.

13. Peters, S.C. and Freedman, D.A. (1987). Comment: Balm for bootstrap confidence intervals. Journal of the American Statistical Association, vol. 82, no. 397, pp. 186-187.

14. Rasmussen, J.L. (1987). Estimating correlation coefficients: Bootstrap and parametric approaches. Psychological Bulletin, vol. 101, no. 1, pp. 136139.

15. Hinkley, D.V. (1988). Bootstrap methods. Journal of the Royal Statistical Society. Series B (Methodological), vol. 50, no. 3, pp. 321-337. 
16. Efron, B. (1988). Bootstrap confidence intervals: good or bad? Psychological Bulletin, vol. 104, no. 2, pp. 293-296.

17. Efron, B. (1983). Estimating the error rate of a prediction rule: Improvement on cross-validation. Journal of the American Statistical Association, vol. 78, no. 382, pp. 316-331.

18. Hall, P. (1986). On the bootstrap and confidence intervals. The Annals of Statistics, vol. 14, pp. 1431-1452.

19. Beran, R.J. (1987). Prepivoting to reduce level error of confidence sets. Biometrika, vol. 74, pp. $457-468$.

20. Loh, W.Y. (1987). Calibrating confidence coefficients. Journal of the American Statistical Association, vol. 82, no. 397, pp. 155-162.

21. Martin, M.A. (1990). On bootstrap iteration for coverage correction in confidence intervals. Journal of the American Statistical Association, vol. 85, no. 412, pp. 1105-1118.

22. McCullough, B.D. and Vinod, H.D. (1998). Implementing the double bootstrap. Computational Economics, vol. 12, pp. 79-95.

23. Letson, D. and McCullough, B.D. (1998). Better confidence intervals: The double bootstrap with no pivot. American Journal of Agricultural Economics, vol. 80, no. 3, pp. 552-559.

24. Chernick, M.R. (2008). Bootstrap Methods: A Guide for Practitioners and Researchers, 2nd ed. John Wiley \& Sons.

25. Arasan, J. and Adam, M.B. (2014). Double bootstrap confidence interval estimates with censored and truncated data. Journal of Modern Applied Statistical Methods, vol. 13, no. 2, pp. 399-419.

26. Prentice, R.L. (1973). Exponential survivals with censoring and explanatory variables. Biometrika, vol. 60 , no. 2 , pp. 279-288.
27. Langlands, A.O., Pocock, S.J., Kerr, G.R. and Gore, S.M. (1979). Long-term survival of patients with breast cancer: a study of the curability of the disease. British Medical Journal, vol. 2, no. 6200, pp. 1247-1251.

28. Byers, R.H.Jr., Morgan, W.M., Darrow, W.W., Doll, L., Jaffe, H.W., Rutherford, G., Hessol, N. and O'Malley, P.M. (1988). Estimating AIDS infection rates in the San Francisco cohort. AIDS (London, England), vol. 2, no. 3, pp. 207-210.

29. Bennett, S. (1983). Log-logistic regression models for survival data. Journal of the Royal Statistical Society. Series C (Applied Statistics), vol. 32, no. 2, pp. 165-171.

30. Cox, D.R. (1970). The analysis of binary data. Methnn \& Co.

31. O’Quigley, J. and Struthers, L. (1982). Survival models based upon the logistic and log-logistic distributions. Computer programs in Biomedicine, vol. 15 , no. 1 , pp. $3-11$.

32. Cox, D.R. and Oakes, D. (1984). Analysis of survival data, ser. Monograph on statistics and applied probability. Chapman and Hall.

33. Gupta, R.C., Akman, O. and Lvin, S. (1999). A study of log-logistic model in survival analysis. Biometrical Journal, vol. 41, no. 4, pp. 431-443.

34. Cox, D.R. and Hinkley, D.V. (1974). Theoretical statistics. CRC Press.

35. Shi, S.G. (1992). Accurate and efficient doublebootstrap confidence limit method. Computational Statistics and Data Analysis, vol. 13, pp. 21-32.

36. Doganaksoy, N. and Schmee, J. (1993). Comparisons of approximate confidence intervals for distributions used in life-data analysis. Technometrics, vol. 35, no. 2, pp. 175-184.

37. Collett, D. (2003). Modelling survival data in medical research. CRC Press. 